\title{
Anisotropic critical phenomena in parabolic geometries: the directed self-avoiding walk
}

\author{
Loïc Turban \\ Laboratoire de Physique du Solide†, Université de Nancy I, BP 239 \\ F-54506 Vandœuvre lés Nancy Cedex, France
}

Received 30 September 1991

\begin{abstract}
The critical behaviour of directed self-avoiding walks is studied on parabolic-like systems with a free boundary at $x= \pm C t^{\alpha}$. Using a scaling argument, $1 / C$ is shown to be a marginal variable when $\alpha=\nu_{\perp} / \nu_{\|}=1 / 2$ i.e. on a parabola. As a consequence the directed walk may display varying local exponents. Such a behaviour is indeed observed for restricted walks. This generalizes a result of Cardy showing that nonuniversal behaviour occurs at corners for isotropic systems.
\end{abstract}

Isotropic systems are known to display nonuniversal critical behaviour at corners $[1-6]$, local exponents varying continuously with the opening angle $\theta$. As shown recently [7], this may be linked to the scale invariance of these shapes in the case of isotropic systems. In parabolic-like geometries where the boundary is located at $X(t)= \pm C t^{\alpha}$ an isotropic change of scale transforms $C$ into $b^{\alpha-1} C$ where $b$ is the dilatation factor. The dimension of $C$ vanishes when $\alpha=1$, i.e. in the corner geometry, then $C=\tan \theta / 2$ is a marginal variable leading to $\theta$-dependent exponents. When $\alpha>1, C$ grows under renormalization and the critical behaviour is that of a flat surface whereas when $\alpha<1, C$ decreases and one gets either a line geometry or a cut, depending on the location of the system relative to its boundary.

These considerations may be extended to the case of anisotropic systems [8] for which the correlation length diverges at the critical point with different critical exponents in the parallel and perpendicular directions. If the $t$-axis of the boundary defined above coincides with the parallel direction of the system with a correlation length exponent $\nu_{\|}=z \nu_{\perp}$, under an anisotropic change of scale [9] with dilatation factors $b_{\|}=b^{z}$ and $b_{\perp}=b, C$ is changed into

$$
C^{\prime}=b^{z \alpha-1} C
$$

and marginal behaviour is obtained when $\alpha=1 / z$.

In the present work, we check these ideas in the case of the directed self-avoiding walk $[8,10]$ for which $z=2$ so that varying exponents are expected inside a parabola. Due to the directedness, one cannot get any boundary effect for a walk outside a parabola. Although in the following we restrict ourselves to the 2D problem, similar results are expected in higher dimensions.

† Unité de Recherche associée au CNRS no 155 
Let us consider a directed walk on a rectangular lattice in the $(x, t)$ plane. At each step with $\Delta t=\tau$ in the time direction, the walker performs a jump $\Delta x= \pm a$ towards one of the two nearest sites with the same probability so that the walk is restricted and may be also considered as a 1D diffusion process. Furthemore the walker is assumed to start at the origin $(x=t=0)$ and remains confined inside a parabolic-like domain which for convenience we consider to be slightly shifted backwards in time

$$
|x(t)|<X(t)=C(t+\eta)^{\alpha}
$$

The number of $N$-step walks starting from the origin and reaching $x=n a$ at time $t=N \tau$ may be written as

$$
\mathcal{N}_{N}(0, n a)=2^{N} P(n a, N \tau)
$$

where the front factor on the right gives the total number of unconfined walks with $N$ steps and $P(x, t)$ gives the probability to reach $x=n a$ without crossing the frontier. This probability satisfies the recursion equation

$$
P(x, t+\tau)=\frac{1}{2}[P(x+a, t)+P(x-a, t)] \quad P(x, 0)=\delta_{x, 0}
$$

with the boundary condition

$$
P(x, t)=0 \quad|x|=X(t)
$$

In the continuum limit $\left(a \ll 1, \tau \ll 1, a^{2} / \tau=1\right)$, one gets the diffusion equation

$$
\frac{\partial P}{\partial t}=\frac{1}{2} \frac{\partial^{2} P}{\partial x^{2}}
$$

together with the time-dependent absorbing boundary condition given in (5).

In a free random walk $\overline{x^{2}(t)}=t$ so that when $\alpha>1 / 2$ the walker no longer sees the frontier when $t \gg t^{*}=C^{2 /(1-2 \alpha)}$ and the probability distribution tends asymptotically to the Gaussian form, namely

$$
P(x, t) \sim \frac{1}{\sqrt{2 \pi t}} \exp \left(-\frac{x^{2}}{2 t}\right) \quad t \gg t^{*}
$$

This is the behaviour expected from equation (1) since $C$ grows under renormalization when $\alpha>1 / 2$ so that the boundary evolves towards a flat surface geometry.

When $\alpha \leq 1 / 2$ the boundary changes the asymptotic behaviour and in order to work with constant boundary conditions, it is convenient to introduce the new variable

$$
y=\frac{x}{(t+\eta)^{\alpha}}
$$

leading to the following equation for $P(y, t)$

$$
(t+\eta)^{2 \alpha} \frac{\partial P}{\partial t}=\frac{1}{2} \frac{\partial^{2} P}{\partial y^{2}}+\alpha y(t+\eta)^{2 \alpha-1} \frac{\partial P}{\partial y}
$$

with absorbing boundary conditions at $y= \pm C$. 
In the appendix, scaling arguments are used to get the form of the probability distribution. Its leading behaviour at long time when $\alpha<1 / 2$ is found to take the form

$$
P(x, t) \simeq \frac{1}{C t^{\alpha}} \exp \left(-\frac{\pi^{2}}{8 C^{2}} \frac{t^{1-2 \alpha}}{1-2 \alpha}\right) \cos \left(\frac{\pi x}{2 C t^{\alpha}}\right)
$$

This asymptotic expression becomes exact in the limit of a strip geometry when $\alpha=0$. A similar stretched exponential behaviour was previously observed in isotropic parabolic systems with relevant boundary effects [7].

In the marginal case $\alpha=1 / 2$ the variables separate in (9) and the problem is exactly solvable. The diffusion equation becomes

$$
(t+\eta) \frac{\partial P}{\partial t}=\frac{1}{2} \frac{\partial^{2} P}{\partial y^{2}}+\frac{y}{2} \frac{\partial P}{\partial y}
$$

and looking for $P$ as a product $\phi(t) \psi(y)$ enables us to write down

$$
\begin{aligned}
& (t+\eta) \frac{\mathrm{d} \phi}{\mathrm{d} t}=-\lambda^{2} \phi \\
& z \frac{\mathrm{d}^{2} \psi}{\mathrm{d} z^{2}}+\left(\frac{1}{2}-z\right) \frac{\mathrm{d} \psi}{\mathrm{d} z}-\lambda^{2} \psi=0
\end{aligned}
$$

where we used the new variable $z=-y^{2} / 2$ in the eigenvalue equation (12b). One recognizes Kummer's equation [11] so that the eigenfunctions are confluent hypergeometric functions. Even solutions ${ }_{1} F_{1}\left(\lambda^{2}, \frac{1}{2} ; z\right)$ must be selected since the initial condition introduces a reflection symmetry with respect to the time axis. These have the following z-expansion

$$
\begin{aligned}
{ }_{1} F_{1}(a, b ; z)=1+\frac{a}{b} z+ & \frac{a(a+1)}{b(b+1)} \frac{z^{2}}{2 !}+\cdots \\
& +\frac{a(a+1)(a+2) \cdots(a+n-1)}{b(b+1)(b+2) \cdots(b+n-1)} \frac{z^{n}}{n !}+\cdots
\end{aligned}
$$

In order to satisfy the boundary conditions, the eigenvalues $\lambda_{m}^{2}$ have to belong to a discrete set which, according to (2) and (8), corresponds to zeros of the confluent hypergeometric function for $z=-C^{2} / 2$. They are solutions of the implicit equation

$$
{ }_{1} F_{1}\left(\lambda_{m}^{2}, \frac{1}{2} ;-\frac{C^{2}}{2}\right)=0
$$

The first differential equation (12a) simply yields

$$
\phi_{m}(t) \sim(t+\eta)^{-\lambda_{m}^{2}}
$$

and putting these together, one may finally write

$$
P(x, t)=\sum_{m} A_{m}(\eta)(t+\eta)^{-\lambda_{m}^{2}} F_{1}\left(\lambda_{m}^{2}, \frac{1}{2} ;-\frac{x^{2}}{2(t+\eta)}\right)
$$




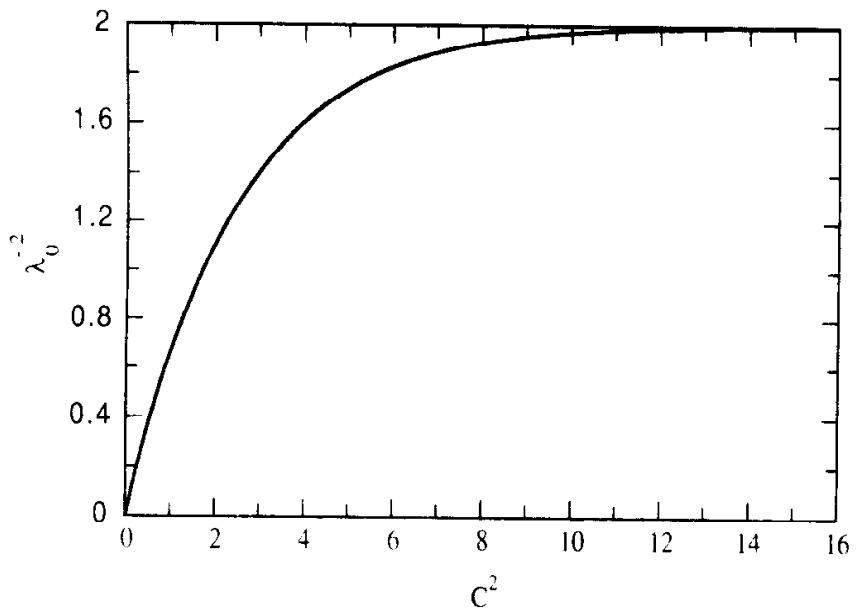

Figure 1. Inverse of the largest eigenvalue of equation (12b) as a function of the square of the marginal parameter $C$.

where the coefficients $A_{m}(\eta)$ have to be chosen to satisfy the initial condition. The asymptotic behaviour is governed by the lowest eigenvalue $\lambda_{0}^{2}$ which was studied numerically (figure 1).

Some analytical results may be obtained only in limiting cases. Let us first consider the strong curvature limit $\varepsilon=C^{2} / 2 \rightarrow 0$. Then, according to (13) and (14) $\lambda_{m}^{2}$ is $O\left(\varepsilon^{-1}\right)$ and introducing $u=C^{2} \lambda_{m}^{2}=O(1)$ in (14), one gets $\cos (\sqrt{2 u})=O(\varepsilon)$ so that the eigenvalue spectrum reads

$$
\lambda_{m}^{2}=\left(m+\frac{1}{2}\right)^{2} \frac{\pi^{2}}{2 C^{2}}+O(1) \quad m=0,1,2 \cdots
$$

The eigenfunctions are obtained in the same way leading to

$$
{ }_{1} F_{1}\left(\lambda_{m}^{2}, \frac{1}{2} ;-\frac{x^{2}}{2(t+\eta)}\right)=\cos \left[\left(m+\frac{1}{2}\right) \frac{\pi x}{C \sqrt{t+\eta}}\right]+O\left(C^{2}\right)
$$

The completness relation

$$
\frac{1}{C \sqrt{\eta}} \sum_{m=0}^{\infty} \cos \left[\left(m+\frac{1}{2}\right) \frac{\pi x}{C \sqrt{\eta}}\right]=\delta(x)
$$

may be used to deduce the coefficients $A_{m}(\eta)$ satisfying the initial condition $P(x, 0)=$ $\delta(x)$ and the probability distribution behaves as

$P(x, t)=\frac{1}{C \sqrt{\eta}} \sum_{m=0}^{\infty}\left(1+\frac{t}{\eta}\right)^{-\left(m+\frac{1}{2}\right)^{2} \frac{\pi^{2}}{2 C^{2}}} \cos \left[\left(m+\frac{1}{2}\right) \frac{\pi x}{C \sqrt{t+\eta}}\right]+O(C)$

the asymptotic behaviour being governed by the first term in the eigenvalue expansion, namely

$$
P(x, t) \sim t^{-\frac{\pi^{2}}{8 C^{2}}} \cos \left(\frac{\pi x}{2 C \sqrt{t}}\right) \quad t \gg 1
$$


This is just the result given by (10) when $\alpha \rightarrow 1 / 2$ if $t^{1-2 \alpha}$ is changed into $t^{1-2 \alpha}-1$ in the exponential, which amounts to modify a constant prefactor, in order to get a meaningfull limit.

Let us now consider the opposite limit. First, when $\mathrm{C}$ is infinite, the boundary condition is satisfied with $\lambda_{0}^{2}=1 / 2$ since [11]

$$
{ }_{1} F_{1}\left(\frac{1}{2}, \frac{1}{2} ; z\right)=\mathrm{e}^{z}
$$

which corresponds to the Gaussian distribution

$$
P(x, t)=\frac{1}{\sqrt{2 \pi(t+\eta)}} \exp \left[-\frac{x^{2}}{2(t+\eta)}\right]
$$

satisfying the initial condition when the limit $\eta \rightarrow 0$ is taken. This is the free diffusion result (7) as expected for a walker starting on a surface which is flat in this limit. When the curvature is weak $(C \gg 1)$ one may use the $z^{-1}$-expansion of the confluent hypergeometric function

${ }_{1} F_{1}(a, b ; z)=\frac{\Gamma(b)}{\Gamma(a)} e^{z} z^{a-b}\left[1+O\left(|z|^{-1}\right)\right]+\frac{\Gamma(b)}{\Gamma(b-a)}(-z)^{-a}\left[1+O\left(|z|^{-1}\right)\right]$

to find out the leading correction to $1 / 2$. After some algebra one gets

$$
\lambda_{0}^{2}=\frac{1}{2}+\frac{C}{\sqrt{2 \pi}} \exp \left(-\frac{C^{2}}{2}\right)[1+O(\varepsilon)]
$$

where $\varepsilon$ is the correction term itself.

Let us finally turn to the evaluation of the critical exponents. Besides the radius of gyration exponents $\nu_{\|}=1$ and $\nu_{\perp}=1 / 2$, in analogy with thermal critical phenomena, one defines a bulk susceptibility exponent $\gamma$ which enters into the asymptotic behaviour of the total number of $N$-step directed walks starting from the origin on an unlimited lattice, $\mathcal{N}_{N} \sim \mu^{N} N^{\gamma-1}$ [8]. Since for the restricted walk, $N=t$ when the time is measured in $\tau$ units, by (3) this can be written as

$$
\mathcal{N}_{N}=2^{N} \int_{-\infty}^{+\infty} P(x, t) \mathrm{d} x=2^{N}
$$

so that $\mu=2$ and $\gamma=1$. Surface exponents may be defined in a system with a straight free surface along the time axis by considering the total number of $N$-step walks starting near the surface at $x=\delta$

$$
\int_{0}^{\infty} \mathcal{N}_{N}(\delta, x) \mathrm{d} x \sim \mu^{N} N^{\gamma_{1}-1}
$$

and the number of $N$-step walks starting and ending near the surface

$$
\mathcal{N}_{N}(\delta, \delta) \sim \mu^{N} N^{\gamma_{11}-1}
$$

Using the Gaussian distribution (7) and the method of images [10], it is easy to check that $\gamma_{1}=1 / 2$ and $\gamma_{11}=-1 / 2$ in agreement with the scaling law $2 \gamma_{1}-\gamma_{11}=\gamma+\nu_{\perp}$ [8]. 
Now, in the parabolic geometry, one may define two new exponents by considering either the total number of $N$-step walks starting near the tip

$$
\int_{-C t^{\alpha}}^{+C t^{\alpha}} \mathcal{N}_{N}(0, x) \mathrm{d} x \sim \mu^{N} N^{\gamma_{0}-1}
$$

or the number of $N$-step walks starting near the tip and ending near the boundary

$$
\mathcal{N}_{N}\left(0, C t^{\alpha}-\delta\right) \sim \mu^{N} N^{\gamma_{01}-1}
$$

Using the analogy with thermal critical phenomena, it may be shown that when $1 / 2 \geq \alpha>0$ these exponents satisfy the scaling law

$$
\gamma_{0}-\gamma_{01}=\gamma_{1}-\gamma_{11}
$$

since the boundary is asymptotically flat and parallel to the time axis. When $\alpha>1 / 2$ one evidently gets $\gamma_{0}=\gamma=1$ and $\gamma_{01}$ remains undefined since at long time the walks cannot reach the surface. In the marginal case, $\alpha=1 / 2$, the leading contribution to $P(x, t)$ in $(16)$ gives

$$
\int_{-C \sqrt{t}}^{+C \sqrt{t}}{ }_{1} F_{1}\left(\lambda_{0}^{2}, \frac{1}{2} ;-\frac{x^{2}}{2 t}\right) \mathrm{d} x \sim t^{1 / 2}
$$

which combines with (3), (16) and (29) to yield

$$
\gamma_{0}=\frac{3}{2}-\lambda_{0}^{2}
$$

The second exponent is obtained in the same way through a first-order expansion of the confluent hypergeometric function. Then, by (14)

$$
{ }_{1} F_{1}\left(\lambda_{0}^{2}, \frac{1}{2} ;-\frac{C^{2}}{2}+\frac{C \delta}{\sqrt{t}}\right) \sim t^{-1 / 2}
$$

and finally

$$
\gamma_{01}=\frac{1}{2}-\lambda_{0}^{2}
$$

follows in agreement with the scaling law (31). When $\alpha<1 / 2$, by (3) and (10), the connective constant $\mu$ is given by

$$
\ln \mu=\ln 2-\lim _{N \rightarrow \infty} \frac{\pi^{2}}{8 C^{2}} \frac{N^{-2 \alpha}}{1-2 \alpha}
$$

so that $\mu=2$ when $\alpha>0$ and due to the exponential decay of the probability distribution, $\gamma_{0}=\gamma_{01}=-\infty$. It follows that when $\mathrm{C}$ varies from zero to infinity, the marginal exponents interpolate between their values below and above $\alpha=1 / 2$. When $\alpha=0$, by (36), the connective constant is changed into

$$
\mu=2 \exp \left(-\frac{\pi^{2}}{8 C^{2}}\right)
$$

and as a consequence of the one-dimensionality of the system $\gamma_{0}=\gamma_{01}=1$. Let us mention that doing the calculation with the transfer matrix technique on a lattice would give $\mu=2 \cos (\pi / 2(C+1))$ instead of (37) but the continuum limit used here is valid for $a \ll 1$ so that both expressions should be compared in the limit $C \gg 1$ where they indeed give the same result. Finally, when $\alpha<0$, the systems shrinks at long time and according to (36) the connective constant vanishes.

The author enjoyed long discussions with Ingo Peschel and Ferenc Iglói about critical phenomena in parabolic geometries. 


\section{Appendix}

As suggested in the introduction the shape of the system may be considered as a perturbation to its critical behaviour in infinite geometry characterized by the scaling field $1 / C$ which, according to (1), may be either relevant, marginal or irrelevant depending on the sign of its scaling dimension $1-z \alpha$. This allows us to write down a scaling ansatz for the probability distribution

$$
P\left(x, t, \frac{1}{C}\right)=b^{-1} P\left(\frac{x}{b}, \frac{t}{b^{z}}, \frac{b^{1-z \alpha}}{C}\right)
$$

where the scaling dimensions are those of the unperturbed fixed point.

With $b=C t^{\alpha}$ and $z=2$ for the directed walk, (A1) translates into

$$
P\left(x, t, \frac{1}{C}\right)=\frac{1}{C t^{\alpha}} f\left(\frac{x}{C t^{\alpha}}, \frac{t^{1-2 \alpha}}{C^{2}}\right)
$$

so that for an irrelevant perturbation, a crossover towards the unperturbed critical behaviour occurs at $t^{*}=C^{2 /(1-2 \alpha)}$ and a comparison with (7) shows that, in this case, the scaling function behaves asymptotically as a Gaussian

$$
f(u, v)=\frac{1}{\sqrt{2 \pi v}} \exp \left(-\frac{u^{2}}{2 v}\right)
$$

Furthermore (A2) can be inserted into (9) to get the asymptotic behaviour of the probability distribution in the case of a relevant perturbation. The scaling function then satisfies

$$
(1-2 \alpha) v \frac{\partial f}{\partial v}-\alpha f=\frac{1}{2} v \frac{\partial^{2} f}{\partial u^{2}}+\alpha u \frac{\partial f}{\partial u}
$$

When $\alpha<1 / 2, v$ grows in time and the leading behaviour is obtained by keeping the two first terms on both sides to get

$$
(1-2 \alpha) v \frac{\partial f}{\partial v}=\frac{1}{2} v \frac{\partial^{2} f}{\partial u^{2}}=-\lambda^{2}
$$

which becomes exact in the strip geometry when $\alpha=0$. With $f \sim \psi(u) \phi(v), \psi$ even in $u$ and vanishing for $u=1$, we find

$$
\begin{aligned}
& f(u, v)=\sum_{m=0}^{\infty} \exp \left(-\frac{\lambda_{m}^{2} v}{1-2 \alpha}\right) \cos \left(\sqrt{2} \lambda_{m} u\right) \\
& \lambda_{m}=\left(m+\frac{1}{2}\right) \frac{\pi}{\sqrt{2}} \quad m=0,1,2 \cdots
\end{aligned}
$$

Together with (A2) this gives a properly normalized exact expression when $\alpha=0$ whereas the term $m=0$ in the eigenvalue expansion provides the leading contribution (10) to the probability distribution. 


\section{References}

[1] Cardy J L 1983 J. Phys. A: Math. Gen. 163617

[2] Barber M N, Peschel I and Pearce PA 1984 J. Stat. Phys. 37497

[3] Cardy J L 1984 Nucl. Phys. B 240514

[4] Bariev R Z 1986 Teor. Mat. Fiz. 69149

[5] Kaiser C and Peschel I 1989 J. Stat. Phys. 54567

[6] Davies B and Peschel I 1991 J. Phys. A: Math. Gen. 241293

[7] Peschel I, Turban L and Iglói F 1991 J. Phys. A: Math. Gen. 24 L1229

[8] Privman V and Švrakić N M 1989 Directed Models of Polymers, Interfaces and Clusters (Lecture Notes in Physics vol 338) (Berlin: Springer)

[9] Binder K and Wang J S 1989 J. Stat. Phys. 5587

[10] Fisher M E 1984 J. Stat. Phys. 34667

[11] Abramowitz M and Stegun I A 1965 Handbook of Mathematical Functions (New York: Dover) p 504 